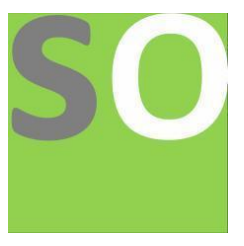

Article title: Tracing to the source of quantization

Authors: Chang-Wei Hu[1]

Affiliations: Beijing Relativity Theory Research Federation, Beijing, China[1]

Orcid ids: 0000-0001-6218-208X[1]

Contact e-mail: huchangwei@sina.cn

License information: This work has been published open access under Creative Commons Attribution License http://creativecommons.org/licenses/by/4.0/, which permits unrestricted use, distribution, and reproduction in any medium, provided the original work is properly cited. Conditions, terms of use and publishing policy can be found at https://www.scienceopen.com/.

Preprint statement: This article is a preprint and has not been peer-reviewed, under consideration and submitted to ScienceOpen Preprints for open peer review.

DOI: 10.14293/S2199-1006.1.SOR-.PPNLNHM.v1

Preprint first posted online: 08 July 2020

Keywords: Quantization, Physical interpretation, Ether, physical vacuum 


\title{
Tracing to the source of quantization
}

\author{
Chang-Wei Hu \\ Beijing Relativity Theory Research Federation, Beijing, China \\ Shanghai Senior Scientist and technician Association, Shanghai, China
}

\begin{abstract}
The physical interpretation of quantum mechanics has always been controversial, which stems from the lack of understanding of vacuum. Vacuum is not empty, and modern physics is describing the world through physical vacuum. Macroscopic vacuum is relatively simple, and relativistic effect is the lens effect of macroscopic vacuum. Micro-vacuum is quite complex, it can be compared to the infinite distribution of micro-lens array or grid. The particles travel through such a vacuum, and there are many possible paths for each step forward, which is just like what path integrals describe. Under the action of micro vacuum, particles will deviate from the positive direction of the starting point to the end point anytime and anywhere. The $\mathrm{i}$ in quantum mechanical equations is a representation of deviation characteristic.
\end{abstract}

Keywords: Quantization, Physical interpretation, Ether (physical vacuum), Departure.

\section{Introduction}

Quantum mechanics is a century old. As one of the two foundations of modern physics, it has achieved remarkable results. However, its achievements are mainly reflected in the quantitative description; The physical interpretation of it is still controversial. In a letter to his friends, Wheeler, a famous physicist, wrote:"December 2000 marks the centenary of the birth of the greatest discovery in physics, the quantum theory. And to celebrate it, I propose a title: Quantum Theory - Our Glory and Our Shame. Why glory, because the development of all branches of physics has the shadow of quantum theory. Why say shame, because a hundred years have passed, we still don't know the origin of quantization." ${ }^{[1]}$ This situation is not only a faint pain of the old problem, but also a germinating of growing point.

The debates among different viewpoints and schools have greatly promoted the development of quantum mechanics. At present, it has been basically confirmed that quantum mechanical effects are independent of the environment, the measurement and the order of experiment, etc. ; the local hidden variable theory of determinism is not established. But this is not to say that quantum mechanics is a complete theoretical system ${ }^{[2]}$.

At present, many scholars devote themselves to conceiving delicate mathematical models. This is often by opening up the dimensions of time and space, looking for new symmetries and so on $\left.^{[3}, 4\right]$. Some people have put forward the theory of multiple worlds ${ }^{[5]}$, and even appeared the movement of "quantum mysticism"[6].

We believe that the main missing of quantum mechanics is its physical mechanism, which should start from its material basis to explore the source of its quantization.

In the macro world, there is a clear distinction between object (the matter with mass) and vacuum. In the microcosmic world, particles and vacuum are closely related: vacuum is the ground state of quantum fields, that is, all quantum fields are in the lowest energy state; all kinds of particles are excited states of quantum field; positive and negative particle pairs can be produced or annihilated in vacuum. This means that particles and vacuum have the same materiality. Based on this fact, we believe that the strange effects of quantum mechanics are the 
manifestation of the interaction between microscopic particles and vacuum.

Quantum mechanics reveals that vacuum is not empty. But its description of the vacuum is drifting and hazy, and it is very complex and difficult to grasp. We find that the macroscopic vacuum is relatively simple, and we can clarify the relationship between the absolute space-time view and the relativistic space-time view. From this circuitous cut, the source problem of quantization may be better solved.

\section{Macroscopic vacuum and space-time view}

We can deduce the Lorentz transformation by using the method of fluid mechanics without the precondition of the invariance of the speed of light. This process contains the logic of the matter having a substantial connection among things. And thus can reveal the physical mechanism of relativity ${ }^{[7,8]}$. The process is quite simple: there is a transformation in fluid mechanics that transforms a compressible fluid into an incompressible fluid, and substituting it into the Galilean transformation, the Lorentz transformation can be obtained. In this process, the corresponding fluid is required to be a superfluid with infinite distribution, and the speed of sound is the speed of light. Such a fluid can only be a physical vacuum, which we call ether and establish the theory of compressibility ether ${ }^{[9]}$. To be more precise, light is the second sound in the superfluid ether.

The hydromechanics derivation of Lorentz transformation connects the Galilean transformation which expresses the absolute space-time view with the Lorentz transformation which expresses the relativistic space-time view; Linking the ether of the vacuum state with the space-time view. It shows that the ether is a compressible superfluid in absolute space-time view and its density is variable; In the relativistic space-time view, the ether is incompressible, that is, its density is invariable, and the speed of light is naturally constant. In fact, the four-dimensional space-time continuum of relativity is the ether, and the relativistic space-time is different from the absolute space-time, which is a space-time of materiality .

The characteristic of absolute space-time view is that the space-time standard is unchanged, but the speed of light will change with the density of ether. Due to the invariance of space-time standard, we can establish a rigid three-dimensional coordinate system and a one-dimensional time axis anywhere, which are the mathematical expressions of a relative space-time in the absolute space-time view.

The feature of relativistic space-time view is that the speed of light is constant, and the space-time standards can vary with the ether density in absolute space-time view. Therefore the absolute space-time view and the relativistic space-time view are two different space-time views in nature. The former does not consider the role of ether, is a pure, real space-time view, because the ether everywhere, quantitatively it will have a certain error; The latter is a material space-time view that observe world through the ether, but it can be consistent with the experimental data, because the experimental process will inevitably be affected by the ether. So the relativistic space-time view, although somewhat distorted by the ether, is a fairly accurate quantitative description. In the case of low speed and weak gravitational field, the change of ether density is small, and its influence is as little as a flat mirror, so the absolute space-time view can be used to describe accurately enough. Under the condition of high speed and strong gravitational field, the change of ether density is greater, and its influence such as concave-convex lens, and thus relativistic effects occur. in other words, relativistic effects are the lens effects of ether, and they can be attributed to the density change effects of ether. 
There are corresponding relationships between the relativistic space-time view and the absolute space-time view:

The relativistic unit length is proportional to the interval between adjacent particles of the ether in absolute space-time.

Relativistic unit time is proportional to the time interval that light passing through the interval of adjacent particles in the ether.

The gravitational field is an ether density field, and the absolute value of the gravitational potential corresponds to the ether density; gravitational field intensity corresponds to the ether density gradient.

The relativistic mass corresponds to the density increment (relative to the ground state) of the ether packet of the object.

The relativistic energy corresponds to the pressure increment (relative to the ground state) of the etheric packet of the object.

Ground state refers to the ether density in the vacuum where an object is located, and different ether densities are different ground states. Then relativistic unit length, unit time, mass and energy can all vary with the ground state.

In the modern quantitative system, the speed of light has become a constant definition speed. This means that the relativistic space-time view is the cornerstone of modern physics. Therefore, the quantitative description of physics must follow the laws of relativity. On the other hand, because the relativistic effect is distorted by the ether, the real space-time view is the absolute space-time view, so we should interpret the physical mechanism of modern physics on the basis of the absolute space-time view.

\section{Quantumization, the lens effects of microscopic ether}

In the view of compressibility ether theory, relativity is the lens effect of macro ether; quantumization is the lens effect of micro ether. A lens, macroscopically, it is smooth; but microscopically, it will be rough. Similarly, the micro-ether will show a certain discreteness, and forming a micro-lens array of infinite distribution. Wilczek, the Nobel Prize winner in physics, believes that the ether in the modern sense is a grid [10], which is infinitely stacked and distributed. Quantitatively, the arrangement of the microlens array or grid is uniform everywhere and isotropic. The microscopic particles travel through such an ether ocean, its own ether field interacting with grids of ether, and there will be many new paths for it to choose with each step forward. So a particle starting from a definite initial state, it is impossible to reach another point along a definite orbit, but can travel through one of countless possible paths to the final state. The various possible paths have respective different probabilities. Its actual path is generally zigzag, but it should be continuous. This path is exactly the same as that described by the path integral. Therefore, in our view, the three equivalent expressions of quantum mechanics, wave mechanics, matrix mechanics and path integral, what can best embody the essence of quantum mechanics is path integral.

Some people think that the motion of microscopic particles is sometimes discontinuous in leaps and bounds, such as quantum jumps. It is true that the image of a lens would appear to be jumping and discontinuous, but following and observing the particle itself, its track should be continuous. Scientists at Yale University carried out an experiment in 2019, and confirm that quantum jump is a continuous process ${ }^{[11]}$. 
Professor Jin Shangnian pointed out that the current theoretical system of quantum mechanics can be summarized as $\mathrm{CMH}+\mathrm{Q}$ mode, $\mathrm{CMH}$ refers to the Hamiltonian theory of classical mechanics, Q refers to some form of quantization hypothesis. The establishment of path integrals is completely in line with this model.

In Hamiltonian Theory of classical mechanics, the action $S$ appears as the integral of Lagrangian $L(\dot{x}, x, t)$ of the system with respect to the time between two nodes $t_{a}, t_{b}$ :

$$
S[x(t)]=\int_{t_{b}}^{t_{a}} L(\dot{x}, x, t) d t
$$

The scheme of Path integral quantization is to assume that every possible path contributes to the phase, where the phase contributed by $x(t)$ is proportional to $\frac{1}{\hbar} \mathrm{S}[x(t)]$ :

$$
\phi[x(t)]=a e^{\frac{i}{\hbar} S[x(t)]}
$$

Then the probability $P(b, a)$ from the initial point $x_{a}$ with time $t_{a}$, to the end point $x_{b}$ with time $t_{b}$ is:

$$
P(b, a)=|\Sigma \phi[x(t)]|^{2}
$$

summation for all $a$ to $b$ paths. In (2)and(3), all physical quantities except $\hbar$ are classical physical quantities.

In wave mechanics, the quantization scheme is as follows: the Hamilton-Jacobi equation in classical mechanics is replaced by the Schrodinger equation in quantum mechanics, and the $\hbar, i$ of characterization system quantization is as coefficient into the Schrodinger equation to realize. In matrix mechanics, the scheme of quantization is that the mechanical quantities are expressed by operators (after given representation, by matrix); the classical Poisson bracket is rewritten as the quantum Poisson bracket; and the $\hbar, i$ of characterization system quantization appears in the operator. In comparison, the quantization scheme of path integral appear simple and intuitive.

\section{Discussion}

Modern physics gives people an abnormal and strange feeling, which comes from people looking at the world through the ether. Because people do not realize that ether is a matter, they unconsciously regard it as a physical space-time, thus forming the special effect of modern physics. On the other hand, the quantitative process is a comparison process among matters, and the quantitative standard must be based on a certain matter. The ether is everywhere, human beings live in it, so it is the best choice to connect the relevant criteria of time and space with the ether density. It is not a coincidence that our quantitative description must conform to relativity, but it would have certain limitations.

The expression of quantum mechanics can not be separated from two special symbolic $\hbar$ and $i$. The role of $\hbar$ in quantum mechanics is to express the particle nature of energy, which is basically clear; the role of $i$ is still unclear.

The plural $A$, as you know, can be written as trigonometric or exponential expression by an angle $\phi$ that deviated from the $\mathrm{X}$-axis: $A=r(\cos \phi+i \sin \phi)$, or $A=r e^{i \phi}$. As a result, we 
believe that $i$ represents the departure of direction in the motion process of microscopic particles, which is the basic reason causing probability or uncertainty. As shown in fig. 1, the positive direction here refers to the straight direction from the starting point a to the end point $b$. In the path integral, this departed angle $\phi=\frac{1}{\hbar} S[x(t)]$, it is a functional that can change at any time.

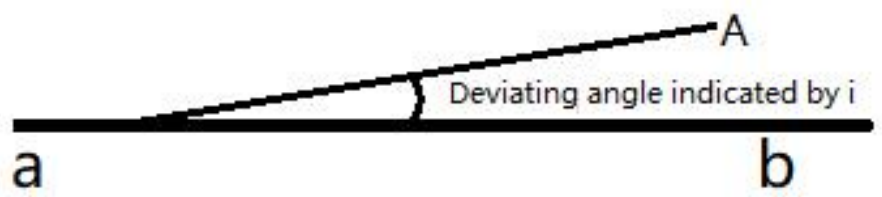

Fig. 1 The angle of departure that can be expressed in the plural

Quantum mechanics is inseparable from mathematics, but mathematics can sometimes complicate simple problems. The collapse of wave function in particle measurement is one of them. In quantum mechanics, a moving particle is accompanied by a "wave of matter", which can collapse instantaneously when it is observed. This can be calculated and explained by the decoherence of quantum entangled states, or by the method of density matrix as the disappearance of all its off-diagonal elements. But they all seem complex and mysterious. In fact, it is generally recognized that the matter wave of a moving particle is a probability wave of where the particle may appear. When a particle is captured by an instrument, its position is determined and its probability disappears. It is simple and clear to explain the so-called collapse of wave function by the disappearance of probability.

\section{References}

[1] Zizhong Zhu, Quantum Mechanics, Tsinghua University Press, pIV（2018）.

[2] Rukang Su, Quantum Mechanics (2nd Edition), Higher Education Press, p471

[3] Guoqiu zhao, Describe Quantum Mechanics in the Dual 4-dimensional Complex Space-Time, Modern physics, 3, 5, pp147-160(2013).

[4] Changpu Sun, New Progress in Fundamental Aspects of Quantum Mechanics, Physics, 30,5, pp310-313(2001).

[5] Lan Ma, A Comparative Study of Quantum Mechanics Explanations from Multiple Horizons, PhD Dissertation of Huazhong University of Science and Technology (2009)

[6] Robert P. Crease, Alfred Scharff Goldhaber, Quantum moment, POSTS\&TELECOM PRESS, pp279-288 (2016).

[7] Chang-Wei Hu. Derivation of the Relativistic Equations from Classical Continuum Mechanics on the Basis of a Macroscopic Vacuum, Physics Essays, Volume 27: Pages 375-379(2014).

[8] Chang-Wei Hu, The Logic Structure Deriving Lorentz Transformation, Scienceopen reprints, 11.13 (2019) . DOI: 10.14293/S2199-1006.1.SOR-.PPBEOMV.v1

[9] Chang-Wei Hu, The Theory of Compressibility Ether, Modern Physics, 7(4): 112-133(2017). 
[10] Frank Wilczek, Lightness of Being:Mass, Ether and Unification of forces, Brockmen Inc.(2009)

[11] Z. K. Minev and other, To catch and reverse a quantum jump midflight, Nature, 570, 200-204(2019). 